\title{
Schütte, Christian, Gesetze am Himmel
}

\section{Gérald Péoux}

\section{OpenEdition \\ Journals}

Édition électronique

URL : http://journals.openedition.org/ifha/2133

DOI : 10.4000/ifha.2133

ISSN : 2198-8943

Éditeur

IFRA - Institut franco-allemand (sciences historiques et sociales)

\section{Référence électronique}

Gérald Péoux, «Schütte, Christian, Gesetze am Himmel », Revue de l'IFHA [En ligne], Date de recension, mis en ligne le 01 janvier 2010, consulté le 22 septembre 2020. URL : http://journals.openedition.org/ ifha/2133 ; DOI : https://doi.org/10.4000/ifha.2133

Ce document a été généré automatiquement le 22 septembre 2020.

(CIFHA 


\title{
Schütte, Christian, Gesetze am Himmel
}

\author{
Gérald Péoux
}

Cet ouvrage a pour objet l'histoire de l'astronomie en Occident. Pour l'auteur, il s'agit de ne pas dissocier les aspects diachronique et épistémologique. Ainsi, refusant la séparation entre ces deux postures, l'auteur progresse-t-il chronologiquement tout en s'interrogeant sans cesse sur le concept de « loi » aux différentes époques et dans les différentes aires géographiques considérées. L'historiographie attribue traditionnellement ce concept au XVIIe siècle et à l'œuvre de Descartes. Or, selon l'auteur, d'autres savants avant le philosophe français avaient déjà affirmé l'existence de lois : Copernic, ce qui est souvent ignoré, ou Roger Bacon au XIIIe siècle qui évoquait les « lois " de la réflexion ou de la réfraction en optique. Il s'agira donc de revisiter l'historiographie en proposant une analyse épistémologique contextualisée des concepts. C'est ce que propose l'auteur en deux grandes parties, d'abord en parcourant les grandes étapes de l'histoire de l'astronomie et en montrant comment les savants des différentes époques ont tenté soit de sauver les phénomènes (grâce à la géométrie) soit de les expliquer en dégageant des lois (en se plaçant du point de vue de la physique). Dans une seconde partie, l'auteur analyse plus finement la recherche de ces lois et leur fondement épistémologique.

Rappelant que la cosmologie grecque fut notamment représentée par le système homocentrique d'Aristote plus tard modifié par les modèles d'Eudoxe et de Ptolémée qui introduisirent un système de cercles (excentriques, déférents et épicycles) et de points (équants), l'auteur montre que l'astronome est libre de recourir à tout artifice mathématique dans la mesure où il « sauve les phénomènes ", en l'occurrence les deux anomalies (variation de la vitesse angulaire et mouvement de rétrogradation des planètes). À l'inverse, l'astronomie arabe se positionne différemment sur le plan épistémologique puisque l'on trouve certains modèles dont la réalité physique est affirmée (comme celui de Tusi qui représente une cosmologie en trois dimensions). Par ailleurs, certains auteurs arabes, comme Alhazen ou Averroès, ont virulemment critiqué le modèle ptoléméen. Pour ce dernier, il est nécessaire de combiner théorie et observation pour élaborer des hypothèses dont la vérification réside dans une 
comparaison avec la physique. Au XIIIe siècle latin, l'astronome continue à sauver les phénomènes. C'est à la Renaissance, avec l'apparition de textes byzantins, que certains auteurs, comme Georg Peuerbach (1421-1461), confèrent aux excentriques et épicycles une dimension matérielle. Au XVIe siècle, l'astronomie entre dans une crise dont l'origine est à attribuer, selon l'auteur, à la difficulté de choisir entre un système physique qui suppose une sélection des causes et un système dont l'objet ne serait que de rendre compte des phénomènes. L'auteur analyse ensuite le débat qui amène Copernic à construire son modèle héliocentrique. Il se positionne dans la discussion historiographique qui oppose ceux pour qui Copernic recherche les causes des mouvements planétaires et ceux pour qui le savant polonais ne cherchait qu'un modèle mathématique.

La seconde partie est consacrée à une discussion plus approfondie sur le concept de loi en philosophie naturelle. Pour certains auteurs, l'idée d'un Dieu tout puissant fait naître le concept de loi de la Nature. Son origine est donc à rechercher dans les racines théologiques et notamment dans les textes de l'Ancien Testament et chez certains théologiens ou philosophes (comme Thomas d'Aquin, Guillaume d'Ockham). Ainsi, s'appuyant sur la lecture des ces derniers philosophes, l'auteur se propose d'éclairer certaines représentations cosmologiques (comme la Cosmosgraphia de Maurolico) en montrant que chez ces savants, la notion de loi est présente et est à rattacher à la divinité. L'auteur poursuit son raisonnement par une analyse de la pensée de Rhéticus, unique élève de Copernic, de Galilée et de Kepler, offrant ainsi un éclairage précis sur une période décisive de l'histoire de sciences.

Gérald Péoux (Observatoire de Paris - SYRTE) 